\title{
Modeling Stochastic Correlated Failures and their Effects on Network Reliability
}

\author{
Mahshid Rahnamay-Naeini*, Jorge E. Pezoa*, Ghady Azar ${ }^{\dagger}$, Nasir Ghani*, and Majeed M. Hayat*‡ \\ *Department of Electrical and Computer Engineering, University of New Mexico, Albuquerque, NM 87131, USA \\ $\dagger$ Electrical and Computer Engineering Department, American University of Beirut, Beirut, Lebanon \\ $\ddagger$ Center for High Technology Materials, University of New Mexico, Albuquerque, NM, USA \\ E-Mail: \{mrahnama, jpezoa, gazar, nghani, hayat\}@ece.unm.edu
}

\begin{abstract}
The physical infrastructure of communication networks is vulnerable to spatially correlated failures arising from various physical stresses such as natural disasters (earthquakes and hurricanes) as well as malicious coordinated attacks using weapons of mass destruction. Some disaster events such as earthquakes and terrorist attacks may occur in more than one location in a short period of time. Hence multiple sets of correlated link failures may occur if more events occurred before the previous set of failed links were repaired. Here, the statistical properties of induced-failure patterns depend upon the spatial interaction among stress centers (e.g., interaction among earthquake or attack locations). This paper presents a stochastic model, based on spatial point processes, for representing stress centers on the geographical plane in order to facilitate the modeling of spatially inhomogeneous and correlated link failures in communication networks. This model is then used to further generate scenarios with inhibition or clustering between stress centers, which enables detailed assessment of vulnerabilities of the network to the level of inhomogeneity and spatial correlation in the stress-event centers. Detailed simulation results are presented to compare network reliability for various scenarios of link failures and to find geographically vulnerable areas of a network as well as worstcase scenarios of stress-events. Overall, this effort will provide some critical knowledge and simulation capabilities for other focus areas of research in network reliability and survivability.
\end{abstract}

Index Terms-Correlated Failures, Communication Networks, Network Stress, Point Processes, Reliability

\section{INTRODUCTION}

The physical infrastructure of backbone communication networks is based on fiber-optic links located on a large geographical plane. As a smaller scale example, a city-scale metropolitan area network is a large communication network that typically spans a metropolitan area geographically. In general, physical infrastructure of communication networks is vulnerable to failures instigated by various physical stresses. At the large-scale these instigators include natural-disaster events (earthquakes and hurricanes), events resulting from coordinated attacks by weapons of mass destruction (WMD) and massive blackouts. At a smaller scale, stress instigators can result from damage due to construction and accidents as well as regional power shortages. Such stresses may occur in any geographic location; however, certain geographical locations can be more prone to host these stresses.

The probability of physical failure for the network components typically depends on both the geographical distance of the components to the center of the event and the intensity of the disaster event. In this sense, geographically correlated failures occur due to geographical proximity of links to the stress centers. Mainly all the papers in this field have used this concept to generate geographically correlated failures (e.g. [1] and [2]). Some disaster events such as earthquakes, terrorist attacks and construction accidents may occur in more than one location in a short period of time. Hence multiple sets of correlated link failures may occur if more events happen before the previous set of failed links were repaired.

Furthermore, the occurrence of some events in a specific geographical region affects the probability of occurrence of other events in other geographical areas. For example, for both tactical and strategic reasons, centers of WMD attacks can exhibit strong correlation. Additionally, earthquakes may also trigger other earthquakes and aftershocks in their vicinities representing another example of correlation between event centers [3]. We emphasize that while event centers - the points where failures are instigated by a stress-are stochastic they can exhibit strong spatial correlations. Therefore, correlated failures in communication networks can arise from correlation amongst the stress-events in addition to geographical proximity to the event centers.

To the best of our knowledge, almost all prior works in modeling correlated failures have considered single-disaster events generating the correlated failures. The recent work in [4] is the only work considering multiple simultaneous disasters. In this paper we develop a mathematical model and a simulation tool to generate and analyze link/node failures due to multiple, stochastic geographic stresses. The presented framework allows us to model various scenarios of external events ranging from totally random locations to spatially correlated event occurrence. Our goals are to (1) gain insight into reliable network design by developing tools that enable simulating various scenarios of stress and failure over the network and (2) identify the geographically vulnerable areas of a network as well as worst-case scenarios of stress-events. Note that reliability of the network clearly depends on the topology as well as the scenario in which the disaster events occur. Overall, this effort will provide some critical inputs for future network reliability and survivability studies.

This paper is organized as follows. Section II presents a survey of the existing work on modeling correlated link failures in communication networks. Section III describes the probabilistic model for correlated link failures based on 
spatial point processes for multiple external stress-events. This model provides us with a tool to assess the reliability and performance of networks in Section IV for various scenarios of events. Finally, conclusions and directions for future work are presented in Section V.

\section{RELATED WORK}

While correlated component failures in communication networks and their effects on reliability have been largely the concern of researchers for a long time, there are very few effective works on modeling such real-world scenarios. In an early work for modeling correlated failures, the authors in [5] considered the dependence between link failures and presented an event-based reliability model. In their model, independent events are used to generate the dependent link failures. This is achieved by defining independent events over sets of components (links), which, for example, share common equipments. Early origins of correlated failures in communication networks were primarily the common equipments between a set of components and the fact that failure of the equipment results in the failure of all the components in the set. Here, the events have certain probabilities of occurrence; however, components in the set sharing the equipment fail with certainty if the event happens. In a similar way, the concept of Shared Risk Link Group (SRLG) has been proposed in [6] in order to address multiple correlated link failures. An SRLG is a set of links sharing a common physical resource (cable, conduit, etc.) and thus a common risk of failure. Later, the authors in [7] used the concept of probabilistic SRLG to address the stochastic correlated component failures; in this model components in an SRLG fail in a probabilistic sense.

In contrast to previously mentioned works, there are other works that have focused on failures within specific geographical regions [2], [8]; such assumption implies that the failed components do not necessarily share the same physical resources [4]. To the best of our knowledge, the main focus of these works are on survivability and reliability analysis of the network in the case of correlated failures and there are few efforts to systematically modeling geographically correlated failures. In [1], a framework is presented to model correlated failures caused by disasters on the networks using vertical cuts; nonetheless, the model is limited to bipartite networks and vertical regional disasters, making it inadequate for modeling stress from WMD attacks and earthquakes. The authors in [2] have modeled disasters or attacks using circular cuts, which are modeled as a disk of radius $r$ centered at the event location; they have assessed the vulnerability of the fiber infrastructures to both circular and vertical cuts. In a similar approach, the authors in [4] used a probabilistic failure model in which components in the vicinity of the disaster (inside the disc) fail with some probability while other components (outside the disc) do not fail. However, using the same probability of failure for all the components inside the disc is not realistic since the effect of events reduces as the distance to the center of the event increases. In contrast to our paper, none of these works have proposed a model for probabilistic occurrence of events in a geographical plane and the interactions between these events.
Correlated failures have been also studied in logical topologies (higher layers of network protocol stack) of networks ( [9] and [10]). In many of these studies, random graphs and percolation theory [11] are common tools to evaluate the effect of random component failures in the network. An example of the source of such correlated failures are logical attacks such as coordinated cyber attacks (e.g., denial of service) affecting higher layer component of the networks such as routers. There are other papers that discuss correlated failures in various contexts ([12] and [13]) other than communication networks. There are also models for temporally correlated failures [14].

Most related to our paper is the work in [4], which considers multiple disasters with circular cuts. Akin to this effort, we have also identified the most vulnerable points of the network by defining various scenarios of events on the network. However our model is different from the mentioned works and also the work presented in [4]. In this paper, we present a model that provides a powerful tool to systematically generate scenarios of multiple disaster events with various level of spatial correlation among their centers in order to capture more accurately the geographical correlation present in real-world scenarios.

\section{PROBABILISTIC MODEL FOR SPATIALLY CORRELATED FAILURES}

In this section we present a probabilistic approach for modeling correlated link failures due to multiple external stress-events in communication networks. In this model we describe a technique for determining the probability of physical damages (failures) for an arbitrary collection of links due to external stress-events with spatially correlated centers. As described earlier, while disaster events are stochastic they can exhibit spatial correlations. Furthermore, there can be spatial inhomogeneity in the concentration of event centers since certain locations are more prone to host the events than others.

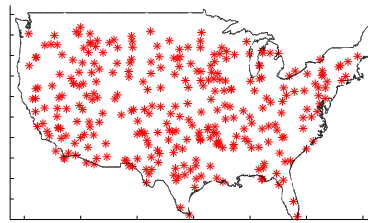

(a)

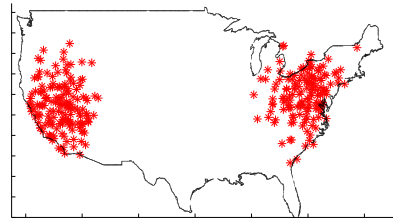

(b)
Fig. 1. Superposition of 50 realizations of a point process each having 5 event centers in the plane. (a) Points resulting from uniform (homogeneous) intensity function; and (b) points resulting from an inhomogeneous intensity function with two regions of high concentration.

Figure 1-a shows an example of a uniform (homogeneous) Poisson point process in the plane. In this case, events can occur anywhere in the plane and without any spatial structure; namely, these patterns are the result of a uniform intensity function (a flat surface over the geographical plane of interest) [15]. In contrast, Fig. 1-b shows an inhomogeneous point process with two concentration locations for events. The inhomogeneity in the concentration of events is affected by a non-constant intensity function representing the probability 
of hosting individual stress-events at each point of the plane. For example, to allow more events to occur in a specific geographical area of interest, we set a peak in the probability intensity surface over the specific area.

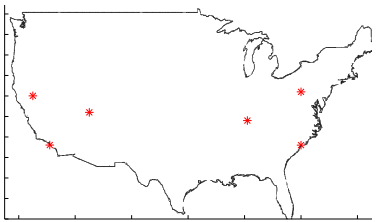

(a)

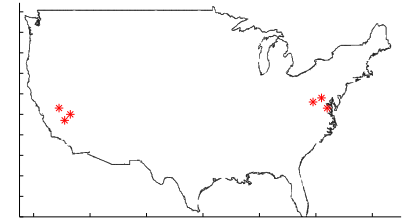

(b)
Fig. 2. Realizations of a Strauss point process with inhomogeneous intensity function and interaction between events for the cases of (a) inhibition and (b) clustering.

Next, we employ an interaction point process model to capture both spatial inhomogeneity and spatial interaction between event centers. The assumption for certain events is that the occurrence of an event in a location affects the likelihood of the occurrence of other events in its vicinity. This effect is called inhibition if the likelihood is reduced and it is called clustering if the likelihood is increased. The Strauss point process is the simplest yet very effective model for inhibition and clustering effects [16]. In the case of inhibition, the probability of the occurrence of points within a fixed radius of an existing point is reduced. When this probability is zero, the inhibition is referred to as hard-core inhibition. In a similar way, in the clustering case the probability of occurrence of events inside of a fixed radius of existing events is enhanced. To simulate the Strauss point process, we used the algorithm presented in [17]. Figure 2 shows realizations of Strauss point process with an inhomogeneous intensity function (Fig. 1-b) and inhibition and clustering effects between centers. Note that the model tends to bias the event centers to be more likely to occur in the vicinity of the two regions of high concentration shown in Fig. 1-b. At the same time, however, there is inhibition and clustering effects between these points defined through the interaction function described bellow. Figure 2a shows a realization with inhibition between event centers, while in Fig. 2-b there are clusters of event centers. Next, we will briefly describe pairwise interaction point-processes [15] of the type used here in order to introduce the key controls of the model in generating various interesting scenarios of events that are relevant to network reliability.

Let $D$ be a bounded subset of the plane. An interaction point process is a $D^{n}$-valued random vector, $\mathbf{X}=\left(X_{1}, X_{2}, \ldots, X_{n}\right)$, which has a probability density of the form

$$
f(\mathbf{x})=z^{-1} \prod_{1 \leq k \leq n} g\left(x_{k}\right) \prod_{1 \leq i \leq j \leq n} \varphi\left(\left\|x_{i}-x_{j}\right\|\right),
$$

where $\mathbf{x}=\left(x_{1}, \ldots, x_{n}\right) \in D^{n}$, and $\|\cdot\|$ is the Euclidean norm in the plane and $z$ is a normalizing constant [15]. The function $g$ represents the intensity function of point distributions. The function $\varphi:[0, \infty) \rightarrow[0, \infty)$ is called the pairwise interaction function; the pairwise behavior of events can be defined through this function. As an example, in the Strauss model [16], $\varphi$ is defined to be equal to a specified value, $c$, for pairs of points that are within the range $R$, and it is set to unity otherwise. The density function of the Strauss process is then

$$
f(\mathbf{x})=z^{-1} c^{S_{\mathbf{x}}(R)},
$$

where for $R>0$ and

$$
S_{\mathbf{x}}(R)=\sum_{1 \leq i \leq j \leq n} I_{(0, R]}\left(\left\|x_{i}-x_{j}\right\|\right)
$$

is the number of pairs of coordinates (points) in $\mathbf{x}$ with pairwise distance less than or equal to $R$, and for any set $A, I_{A}(a)=1$ whenever $a \in A$ and $I_{A}(a)=0$ otherwise. The $R$ parameter in the Strauss model controls the range of interactions between pairs of points. Furthermore, based on the density function of the Strauss model, a value of $c$ greater than 1 results in clustering and a value of $c$ less than 1 results in inhibition. Note that when $c=1$ we obtain the totally random (Poisson) scenario of events in the geographical plane.

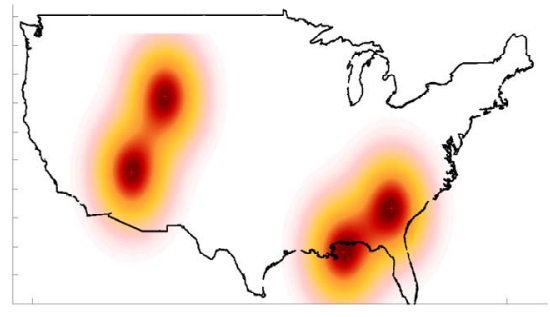

Fig. 3. A representative $C L_{p d}$ corresponding to a sample realization of 4 event locations.

Next, we define the conditional likelihood function of physical damage, $C L_{p d}(\mathbf{y}), \mathbf{y} \in \mathbb{R}^{2}$, for all the points over the geographical plane. We define the probability that a network link, $e$, fails after the occurrence of a disaster event to be $\max _{y \in e}\left(C L_{p d}(y)\right)$. Specifically, we define the intensity of the damage at points of the plane using Gaussian functions placed atop each event center. The Gaussian function is a convenient and appropriate function to model the range and intensity of the disaster events over the geographical plane because of its symmetric bell-curve shape that quickly falls off towards plus/minus infinity. The variance of the Gaussian function controls the range and intensity of the disaster event. The Gaussian functions on top of multiple events are summed up and normalized (to unity) to yield $C L_{p d}(\cdot)$. Aggregating the Gaussian functions also captures the net result of adding the effects of multiple individual disaster events as the Gaussian functions overlap.

Figure 3 shows a representative $C L_{p d}$ corresponding to a sample realization of four event locations with the Gaussian functions atop each event. Note that conditional on a particular realization of $C L_{p d}$, the physical failures of links (in any collection of links) are independent; however, correlation between physical damage is inherited from the correlation in the event centers associated with $C L_{p d}$ and the geographical proximity of the links to the event centers. The $c$ and $R$ parameters of the Strauss model as well as the variance of the Gaussian function enables us to capture scenarios of events with various spatial correlations among their centers with nonuniform spatial intensity in a plane of interest. As a result, we have an effective tool to simulate desirable scenarios of spatial stress-events on the geographical plane to assess the reliability and efficiency of the network in the case of various stress 
scenarios. In the next section we use this model to find the most vulnerable parts of the network and identify the worstcase scenarios of stress-event occurrences.

\section{SIMULATION RESULTS}

In this section the reliability and efficiency of communication networks in the presence of geographically correlated failures due to multiple disaster events are evaluated using a Monte-Carlo (MC) simulation analysis applied to the analytical model for link failures we described earlier. Specifically, a MC simulator for generating correlated failures based on the model presented in Section III was developed in MATLAB. The model presented in Section III provides a powerful tool for randomly locating disaster events on the geographical plane with various interesting scenarios and consequently different statistical characteristics of failures on the network. Evaluations of network reliability and efficiency are presented here for different scenarios of interest for the spatial distribution of the stress centers. In our simulations, we have considered the topology of a fiber backbone operated by a major network provider in the U.S. [18]. As described earlier the model enables us to control the clustering and inhibition characteristics of the disaster events through the selection of the $c$ and $R$ parameters of the Strauss model. It also enables the control of the severity of the events (how strong is the disaster event in affecting the network components and the range of their effect) through the selection of the variance of the Gaussian functions. For a larger intensity of disaster events, a larger variance for the Gaussian function is used.

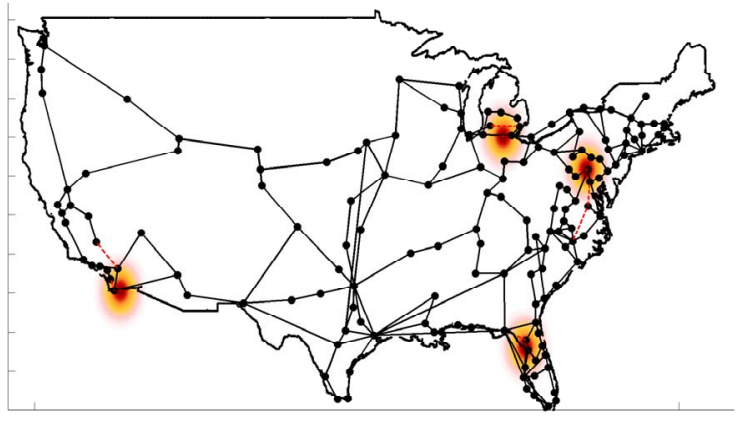

Fig. 4. A random sample of stress-event centers with the Strauss settings of $c=0.3$ and $R=500$, and the variance of Gaussian function set to 600 .

We shall define four scenarios of interest for the locations of stress-events and evaluate network reliability in these four scenarios. In the first scenario we use the inhibition setting in the Strauss model so as to generate random realizations of event centers that are likely to be far from each other. In this scenario the probability of the occurrence of events within a fixed radius of an existing point is reduced. We define the radius to be 500 miles ( $R_{1}=500$ miles), the $c$ parameter to be 0.3 and the variance of the Gaussian functions is set to 600 . A random sample of this scenario is shown in Fig. 4.

The rest of the scenarios are based on clustering mode in the Strauss model with the cluster radius used as a free parameter. In these scenarios, we assume that the probability of events happening farther than $R$ miles is reduced. For each scenario we set the maximum distance between events to $R_{2}=200$, $R_{3}=100$, and $R_{4}=20$ miles, respectively, and the $c$ parameter for all these scenarios is set to 80. Random samples of each of these scenarios are presented in Figs. 5, 6 and 7. Note that in the sample of the Strauss model corresponding to the fourth scenario, events are not farther than 20 miles apart; therefore, stress centers are closer than those in the remaining clustering scenarios. In all the simulations of this section we assume four number of disaster events; however, any number of events can be assumed. For comparison, we also consider the independent link failure scenario without the use of external events to represent the case of totally uncorrelated link failures in the network. (In the latter scenario we do not follow the event-center approach to insure that there is no correlation in the failures.) Next, we present our predictions of the effect of the aforementioned failure scenarios on network reliability and efficiency.

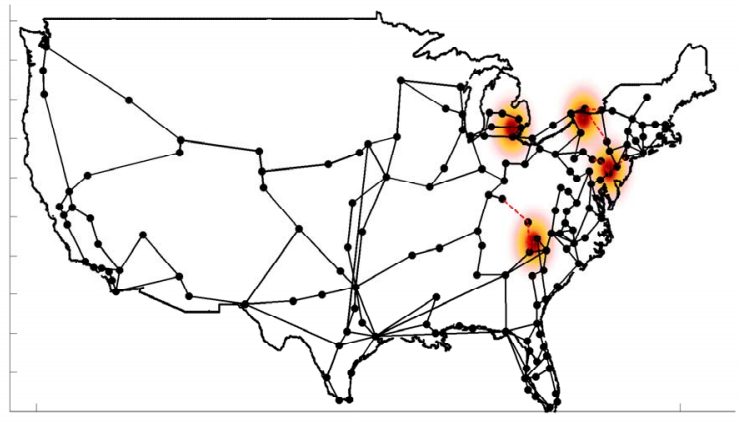

Fig. 5. A random sample of stress-event centers with the Strauss settings of $c=80$ and $R=200$, and the variance of Gaussian function set to 600 .

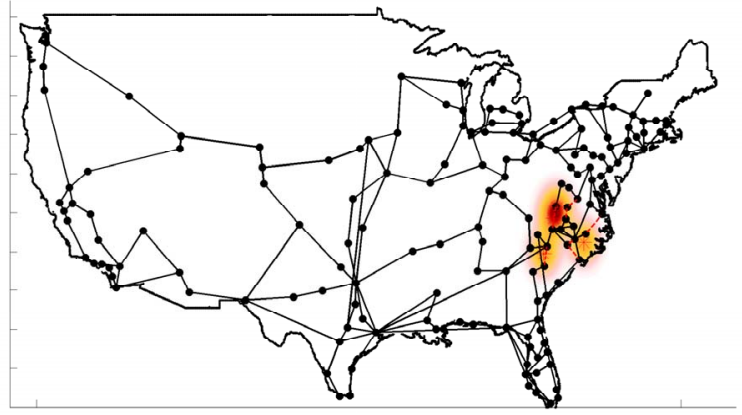

Fig. 6. A random sample of event centers with the Strauss settings of $c=80$ and $R=100$, and the variance of Gaussian function set to 600 .

We have evaluated the reliability of the network based on two metrics: the average two-terminal reliability (ATTR) [4], which is a measure of global connectivity of the network, and the connectivity probability, which is the fraction of the number of times the network remains connected over all runs of the simulations. In Fig. 8, the ATTR values for the four mentioned scenarios and the independent link failure case are presented for different values of the stress effect represented by the variance of the Gaussian functions. For each scenario we have generated 500 random samples and the results presented 


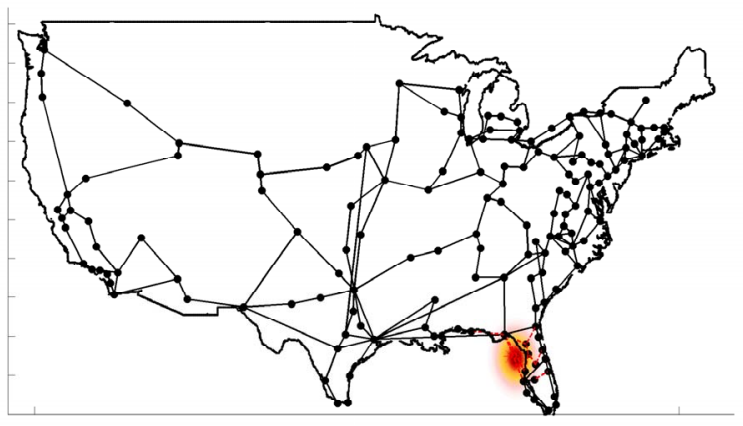

Fig. 7. A random sample of stress-event centers with the Strauss settings of $c=80$ and $R=20$, and the variance of Gaussian function set to 600 .

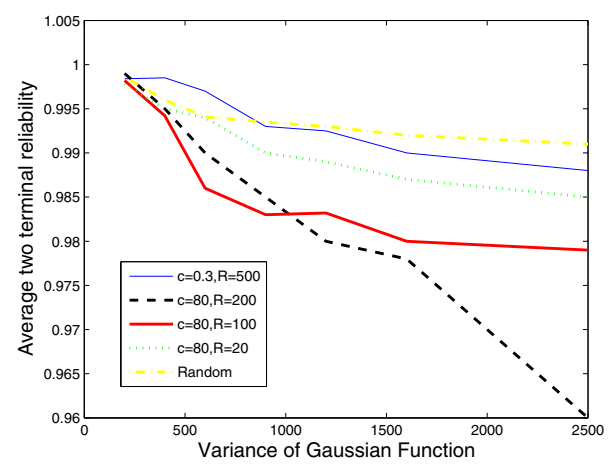

Fig. 8. Average two-terminal reliability.

correspond to averages over all 500 samples. (Note that for the independent link failure scenario, we have calculated the average number of failed links in other four scenarios for each variance value and then generated the same number of failures randomly among the links.) Based on Fig. 8, in

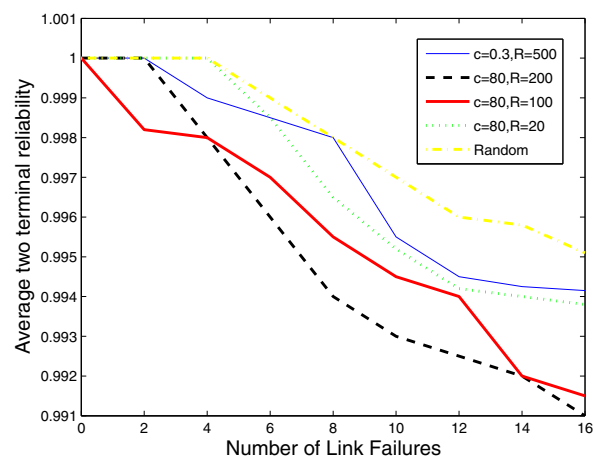

Fig. 9. Average two-terminal reliability.

the first failure scenario the ATTR is higher than those for other scenarios except for the independent link failure case. For small values of the variance of the Gaussian functions, the correlation among failures due to geographical proximity decreases. Moreover, since in the first scenario the event centers do not occur closer than $R_{1}$ of each other, the ATTR value is higher for smaller variances compared to independent link failure case. However, as effect-range or intensity of the disaster increases, the correlation between link failures due to geographical proximity increases, which, in turn, causes network reliability to decrease compared with independent link failure scenario. The other interesting observation in Fig. 8 is that network reliability is less affected in the scenarios with smaller cluster radius than that in the larger clusters. Note that size of clusters assumed to be less than 20 percent of the size of the geographical plane over which the network is expanded.

Figure 9 shows the ATTR value for the samples with the same number of failures and for the variance of the Gaussian functions set to 1200 . In this case, we have taken the average of ATTR over all the realizations with the same number of failures for the four aforementioned scenarios. As such, this plot shows that if we have the same number of failures in the network with different characteristics, they will have different effects on the network reliability. We can conclude based on these two plots that the clustering scenarios with larger effectrange, namely the second scenario $\left(R_{2}=200\right)$, affect the ATTR value more significantly than the other scenarios. As before, the independent random link failure case affects the reliability less severely than other scenarios because of the lack of geographical correlation between the failures.

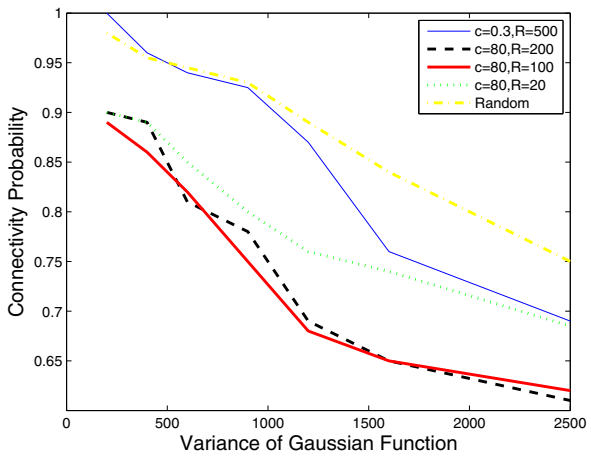

Fig. 10. Connectivity probability.

Figure 10 presents the connectivity probability of the network for the different scenarios based on the variance of the Gaussian functions. Note that the clustering scenarios have lower connectivity probability than other scenarios. This is because the geographical correlation that is present amongst stress centers results in substantial geographically correlated link failures, thereby lowering reliability.

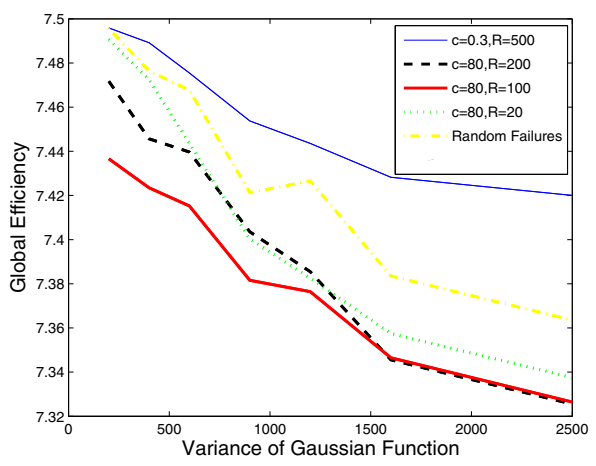

Fig. 11. Global efficiency of the network.

In studying the network efficiency, we have adopted the global efficiency metric defined in [19]. The results of the global efficiency of the network are presented in Fig. 11. Note the similarity of the behavior of the efficiency metric in this figure compared to the ATTR metric shown in Fig. 9. 
Finally, we have also conducted a study on the most vulnerable regions of the network and the worst-case scenario of the link failures. To do this, we tested 2000 random samples of each scenario with four number of disaster events and searched for the stress-distribution scenario that resulted in the least network reliability amongst the four stress-event center scenarios. In this simulation, we fixed the intensity of the stress-event occurrence to 600. The "vulnerable" regions are identified with circles in Fig. 12. These regions are nearly matched with the results presented by Agarwal et al. [4], which did not use a stochastic approach for the distribution of stressevent centers but rather used a greedy heuristic approach that finds the vulnerable points of the network for two simultaneous events. Furthermore, the worst-case scenario for the four number of the disaster events is the second scenario, which is a clustering scenario with a radius of 200 miles.

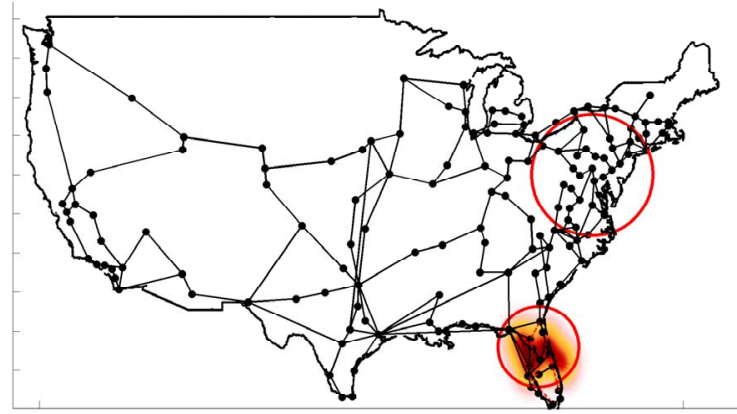

Fig. 12. Vulnerable points of the network assuming a variance of 600 for the Gaussian functions and a random sample of the third stress-event-center scenario.

\section{CONCLUSIONS}

The physical infrastructure of communication networks is increasingly vulnerable to geographically correlated failures. Any systematic assessment of network reliability in the presence of correlated link failures requires a stochastic model of such component failures. Along these lines, in this paper we have undertaken a novel probabilistic framework, based on interaction point processes, for modeling correlated link failures in networks of arbitrary topologies due to multiple external stresses. We addressed the problem of modeling random stress-events that may occur in a short period of time and with the presence of interaction between their locations. This model is capable of capturing correlation due to interaction between stress-event centers as well as geographical proximity of components to the stress-event centers. The model enables generating random scenarios of stress locations with inhibition and clustering characteristics of interest. Based on the evaluation of the reliability using the generated link failures we conclude that correlated failures can cause a more drastic degradation in the reliability than that inflicted by random yet independent failures. We have also been able to identify the vulnerable regions of the network and the worst-case scenarios of stress-event locations. Using these insights, future efforts can look at developing improved network design and recovery strategies in the case of correlated failure scenarios.

\section{ACKNOWLEDGMENT}

This work was supported by the Defense Threat Reduction Agency (Combating WMD Basic Research Program).

\section{REFERENCES}

[1] S. Neumayer, G. Zussman, R. Cohen, and E.Modiano, "Assessing the impact of geographically correlated network failures," in Proc. IEEE MILCOM, 2008.

[2] S. Neumayer, G. Zussman, R. Cohen, and E. Modiano, "Assessing the vulnerability of the fiber infrastructure to disasters," in Proc. IEEE INFOCOM, 2009.

[3] L. Holden, S. Sannan, and H. Bungum, "A stochastic marked point process model for earthquakes," Natural Hazards \& Earth System Sci., vol. 3, pp. 95-101, 2003.

[4] P. Agarwal, A. Efrat, S. Ganjugunte, D. Hay, S. Sankararaman, and G. Zussman, "Network vulnerability to single, multiple, and probabilistic physical attacks," in Proc. IEEE MILCOM, 2010.

[5] V. Latora and M. Marchiori, "Reliability modeling and analysis of communication networks with dependent failures," IEEE Transactions on Communications, vol. 34, no. 1, pp. 82-84, 1986.

[6] D. Papadimitriou et al., "Inference of shared risk link groups." [Online]. Available: http://www3.tools.ietf.org/html/draft-many-inference-srlg-02

[7] H. Lee and E. Modiano, "Diverse routing in networks with probabilistic failures," in Proc. IEEE INFOCOM, 2009.

[8] P. Erdös and A. Rényi, "Some generalized max-flow min-cut problems in the plane," Mathematics of Operations Research, vol. 16, no. 2, pp. 310-333, 1991

[9] D. Magoni, "Tearing down the internet," Selected Areas in Communications, IEEE Journal on, vol. 21, no. 6, pp. 949 - 960, Aug. 2003.

[10] R. Church, M. Scaparra, and R. Middleton, "Identifying critical infrastructure: The median and covering facility interdiction problems," Annals of the Association of American Geographers, vol. 94, no. 3, pp. $491-$ 502, Aug. 2004.

[11] Z. Kong and E. Yeh, "Resilience to degree-dependent and cascading node failures in random geometric networks," Information Theory, IEEE Transactions on, vol. 56, no. 11, pp. 5533-5546, 2010.

[12] K. Goseva-Popstojanova and K. Trivedi, "Failure correlation in software reliability models," IEEE Trans. Reliability, vol. 49, pp. 37-48, 2000.

[13] Y.-S. Dai, G. Levitin, and K. Trivedi, "Performance and reliability of tree-structured grid services considering data dependence and failure correlation," IEEE Trans. Computers, vol. 56, pp. 925-936, 2007.

[14] M. Gallet, N. Yigitbasi, B. Javadi, D. Kondo, A. Iosup, and D. Epema, "A model for space-correlated failures in large-scale distributed systems," in Euro-Par 2010 - Parallel Processing, ser. Lecture Notes in Computer Science, P. DAmbra, M. Guarracino, and D. Talia, Eds. Springer Berlin / Heidelberg, 2010, vol. 6271, pp. 88-100.

[15] D. Daley and D. Vere-Jones, An Introduction to the Theory of Point Processes, 2nd ed. Springer, 2008.

[16] D. Strauss, "A model for clustering," Biometrika, vol. 62, pp. 467-475, 1975.

[17] B. D. Ripley, "Algorithm as 137: Simulating spatial patterns: Dependent samples from a multivariate density," Journal of the Royal Statistical Society. Series C (Applied Statistics), vol. 28, pp. 109-112, 1979.

[18] "Level 3 communications, network map." [Online]. Available: http://www.level3.com/interacts/map.html

[19] P. Crucitti, V. Latora, M. Marchiori, and A. Rapisarda, "Efficiency of scale-free networks: error and attack tolerance," Physica A: Statistical Mechanics and its Applications, vol. 320, pp. 622-642, 2003. 